\title{
METRIC TRANSFORMS AND EUCLIDEAN EMBEDDINGS
}

\author{
M. DEZA AND H. MAEHARA
}

\begin{abstract}
It is proved that if $0 \leq c \leq 0.72 / n$ then for any $n$-point metric space $(X, d)$, the metric space $\left(X, d^{c}\right)$ is isometrically embeddable into a Euclidean space. For 6-point metric space, $c=\frac{1}{2} \log _{2} \frac{3}{2}$ is the largest exponent that guarantees the existence of isometric embeddings into a Euclidean space. Such largest exponent is also determined for all $n$-point graphs with "truncated distance".
\end{abstract}

\section{INTRODUCTION}

One of the fundamental problems in distance geometry is the isometric embedding problem, that is, to determine conditions for metric spaces to be isometrically embeddable in a given class of spaces, say $l_{p}$-spaces (e.g. $[1-5,8,11,12$, $18,23])$. For finite metric spaces, there also arises the minimum dimensional embedding problem, that is, to decide the minimum dimension $m=m_{p}(n)$ such that any $n$-point subset of an $l_{p}$-space can be isometrically embedded in $l_{p}^{m}$. Except for the case $p=2$ (in this case, $m_{2}(n)=n-1$ ), the problem is not trivial. For some bounds on $m_{p}(n), p \neq 2$, see e.g. Ball [6], Witsenhausen [24].

In this paper, we consider deforming the distance function of a metric space so that the resulting space can be embedded in a certain space, say $l_{2}$. This problem has its origin in the 1930s (e.g. $[7,16,19,22])$.

Throughout this paper, $X$ stands for a finite metric space and $X_{n}$ stands for an $n$-point metric space. The distance between two points $x, y$ of a metric space is denoted by the juxtaposition $x y$. If $X$ is isometric to a subset of a Euclidean space, then $X$ is said to be Euclidean. Let $F(t)$ be a continuous, monotone increasing, concave function of $t \geq 0$ with $F(0)=0$. Then, replacing the distance $x y$ in $X$ by $F(x y)$, we have another metric space with the same point set as $X$. This new metric space is called the metric transform of $X$ by $F(t)$, and it is denoted by the symbol $F(X)$.

Blumenthal [8, p. 131] proved that if $0<c \leq \frac{1}{2}$ then the metric transform $\left(X_{4}\right)^{c}$ by $F(t)=t^{c}$ is Euclidean. Further, he showed that $c=\frac{1}{2}$ is the largest exponent that guarantees $\left(X_{4}\right)^{c}$ to be Euclidean for all $X_{4}$. We are going to

Received by the editors May 12, 1988.

1980 Mathematics Subject Classification (1985 Revision). Primary 51K05, 51M05; Secondary $05 \mathrm{C} 10$. 
consider similar problems for $X_{n}, n>4$, in connection with "hypermetric" spaces (e.g. $[3,11,13])$.

We will prove that if $0<c \leq 0.7213 \ldots / n$, then $\left(X_{n}\right)^{c}$ is always Euclidean. In particular, $c=\frac{1}{2} \log _{2} \frac{3}{2}=0.2924 \ldots$ is the largest exponent that guarantees $\left(X_{6}\right)^{c}$ to be Euclidean.

The vertices of a connected graph $G$ constitute a metric space with the shortest path distance. We denote this metric space by the same letter $G$. Winkler [23] gave a characterization of the connected graphs $G$ whose metric transforms $(G)^{1 / 2}$ are Euclidean.

For a graph $G$, define the truncated distance between two vertices $x, y$ of $G$ by

$$
x y= \begin{cases}0 & \text { if } x=y \\ 1 & \text { if } x \text { and } y \text { are adjacent } \\ 2 & \text { otherwise. }\end{cases}
$$

The metric space consisting of the vertices of $G$ with truncated distance is denoted by $G_{T}$. If $G$ is a graph with diameter $\leq 2$, then $G$ and $G_{T}$ are the same space. Isometric embeddings of $G_{T}$ into Hamming hypercubes and $l_{1}$ were considered in $[2,3]$.

We will prove that $c_{n}$, the largest $c$ such that $\left(G_{T}\right)^{c}$ are Euclidean for all $n$-point graphs $G$, is given by

$$
c_{n}= \begin{cases}\frac{1}{2} \log _{2}(k /(k-1)) & \text { if } n=2 k, \\ \frac{1}{2} \log _{2}\left(2 k(k+1) /\left(2 k^{2}-1\right)\right) & \text { if } n=2 k+1 .\end{cases}
$$

\section{Preliminaries}

A space $X$ is said to be hypermetric if

$$
\sum_{x \in X} a_{x}=1 \Rightarrow \sum_{x, y \in X} a_{x} a_{y}(x y) \leq 0
$$

where $a_{x}$ are integers. A space $X$ is of negative type if the above holds with 1 replaced by 0 . Schoenberg [18] proved that $(X)^{1 / 2}$ is Euclidean if and only if $X$ is of negative type. This characterization goes back to Cayley, but was, first stated in this form by Schoenberg in the 1930s. A space $X$ is said to be $l_{1}$-embeddable if there is an isometric embedding of $X$ into $R^{n}$ with the $l_{1}$-metric, i.e. the metric

$$
d(\bar{x}, \bar{y})=\left|x_{1}-y_{1}\right|+\cdots+\left|x_{n}-y_{n}\right| .
$$

A metric space is called ultrametric (see e.g. [1]) if it satisfies $x z \leq \max (x y, y z)$ for any three points $x, y, z$. In Maehara [15], an ultrametric space $X$ is called an irreducible space, because any metric transform of $X$ requires $(|X|-1)$ dimensional Euclidean space for an isometric embedding. In particular, [15, Theorem 7] implies that ultrametric spaces are Euclidean (see also [1, Theorem 6.7]). 
There is the following hierarchy in finite metric spaces (see, e.g. $[2-4,10-13$, 15, 17. 23]):

$$
\begin{aligned}
& X \text { is ultrametric } \\
& \Rightarrow X \text { is Euclidean } \\
& \Rightarrow X \text { is } l_{1} \text {-embeddable } \\
& \Rightarrow X \text { is hypermetric } \\
& \Rightarrow X \text { is of negative type } \\
& \Rightarrow \text { The distance matrix of } X \text { has only one positive eigenvalue. }
\end{aligned}
$$

The conditions for hypermetricity and for negative type can be described by the polygonal inequalities. A space $X$ is said to be $(2 N+\varepsilon)$-gonal if the $(2 N+\varepsilon)$-gonal inequality

$$
\sum_{i<j} x_{i} x_{j}+\sum_{i<j} y_{i} y_{j} \leq \sum_{i, j} x_{i} y_{j}
$$

holds for any $(2 N+\varepsilon)$-sequence of points of $X$,

$$
x_{1}, \ldots, x_{N}, y_{1}, \ldots, y_{N+\varepsilon},
$$

where $\varepsilon=0$ or 1 . These $2 N+\varepsilon$ points are not necessarily different. The 3gonal inequality is just the triangle inequality. Then a space $X$ is hypermetric if and only if it is $(2 N+1)$-gonal for all $N \geq 1$, while $X$ is of negative type if and only if it is $2 N$-gonal for all $N \geq 1$ (e.g. [3]).

In this paper the function $\log _{2}(1+1 / s)$ appears frequently, so we denote this function by $\gamma$ :

$$
\gamma(s)=\log _{2}(1+1 / s) \text {. }
$$

First we prove two lemmas.

Lemma 1. Suppose that $0<c \leq \gamma(s), s>1$. Then, in the metric transform $(X)^{c}, x y \leq y z$ implies that

$$
x z \leq(x y) / s+y z .
$$

Proof. Denote the distance in the space $X$ by $\overline{x y}$. Then $x y=(\overline{x y})^{c}$ by definition. Since $\overline{x y}+\overline{y z} \geq \overline{x z}$, it is enough to show that

$$
(\overline{x y})^{c} / s+(\overline{y z})^{c}-(\overline{x y}+\overline{y z})^{c} \geq 0 .
$$

Dividing the left-hand side by $(\overline{x y})^{c}$ and letting $t=(\overline{y z}) /(\overline{x y})$, we have

$$
f(t):=1 / s+t^{c}-(1+t)^{c} .
$$

Thus it remains only to show that $f(t) \geq 0$ for $t \geq 1$. Now, since the condition $0<c \leq \gamma(s)$ implies that $(1+1 / s) \geq 2^{c}$, we have $f(1) \geq 0$. And since $c<1$, we have

$$
f^{\prime}(t)=c t^{c-1}-c(1+t)^{c-1}>0 \quad \text { for } t \geq 1 .
$$

Therefore $f(t) \geq 0$ for $t \geq 1$. 
Let $(2)$ be a $(2 N+1)$-sequence of points of $(X)^{c}$. By changing suffixes if necessary, we may assume that

$$
x_{k} y_{k} \leq x_{i} y_{j} \quad \text { for } i \geq k, \quad j \geq k, \quad k=1, \ldots, N
$$

holds in the sequence (2). If $i<k$ then by (3) and Lemma 1 ,

$$
\begin{aligned}
& x_{i} x_{k} \leq\left(x_{i} y_{i}\right) / s+x_{k} y_{i} \leq\left(x_{k} y_{k}\right) / s+x_{k} y_{i}, \\
& y_{i} y_{k} \leq\left(x_{i} y_{i}\right) / s+x_{i} y_{k} \leq\left(x_{k} y_{k}\right) / s+x_{i} y_{k} .
\end{aligned}
$$

Hence we have the following lemma.

Lemma 2. Suppose that $0<c \leq \gamma(s)$ and $k \leq N$. If $i<k<j$, then

$$
\begin{aligned}
& x_{i} x_{k} \leq\left(x_{k} y_{k}\right) / s+x_{k} y_{i}, \\
& x_{j} x_{k} \leq\left(x_{k} y_{k}\right) / s+x_{j} y_{k}, \\
& y_{i} y_{k} \leq\left(x_{k} y_{k}\right) / s+x_{i} y_{k}, \\
& y_{j} y_{k} \leq\left(x_{k} y_{k}\right) / s+x_{k} y_{j} .
\end{aligned}
$$

Therefore, for any $i, k \leq N$,

$$
x_{i} x_{k}+y_{i} y_{k} \leq 2\left(x_{k} y_{k}\right) / s+x_{i} y_{k}+x_{k} y_{i} \text {. }
$$

\section{THE POLYGONAL INEQUALITIES}

Theorem 1. If $0<c \leq \gamma(N)$, then the metric transform $(X)^{c}$ is $M$-gonal for all $M \leq 2 N+2$, and if $c>\gamma(N)$ then there is a space $X$ such that $(X)^{c}$ is not $(2 N+2)$-gonal.

Proof. Suppose that $0<c \leq \gamma(N)$. It is known that if a metric space is $(2 N+1)$-gonal, then it is $M$-gonal for all $M \leq 2 N+2$ (see e.g. [11]). Hence we show that $(X)^{c}$ is $(2 N+1)$-gonal.

Let $(2)$ be a $(2 N+1)$-sequence taken from $(X)^{c}$. We may suppose that (3) holds for this sequence. Fix $k<N+1$ and sum (4) over $i \neq k$ to obtain

$$
\begin{aligned}
\sum_{i=1}^{N}\left(x_{i} x_{k}+y_{i} y_{k}\right) \leq & \sum_{i=1}^{N}\left(x_{i} y_{k}+x_{k} y_{i}\right) \\
& -2\left(x_{k} y_{k}\right)+2(N-1)\left(x_{k} y_{k}\right) / N .
\end{aligned}
$$

Summing over $k<N+1$ gives

$$
\sum_{k=1}^{N} \sum_{i=1}^{N}\left(x_{i} x_{k}+y_{i} y_{k}\right) \leq \sum_{k=1}^{N} \sum_{i=1}^{N}\left(x_{i} y_{k}+x_{k} y_{i}\right)-2 \sum_{k=1}^{N}\left(x_{k} y_{k}\right) / N .
$$

Dividing this inequality by 2 , and adding the inequality

$$
\sum_{k=1}^{N} y_{k} y_{N+1} \leq \sum_{k=1}^{N}\left\{\left(x_{k} y_{k}\right) / N+x_{k} y_{N+1}\right\}
$$


we have

$$
\sum_{i<j} x_{i} x_{j}+\sum_{i<j} y_{i} y_{j} \leq \sum_{i=1}^{N} \sum_{k=1}^{N} x_{i} y_{k}+\sum_{k=1}^{N} x_{k} y_{N+1}=\sum_{i, j} x_{i} y_{j} .
$$

This is (1), proving the first part of the theorem. For the second part, consider the complete bipartite graph $K(N+1, N+1)$ with vertex classes $\left\{x_{1}, \ldots, x_{N+1}\right\}$ and $\left\{y_{1}, \ldots, y_{N+1}\right\}$. Then all the vertices of $K(N+1, N+1)$ constitute a metric space $X$ by the usual graph distance. Suppose $c>\gamma(N)=\log _{2}(1+1 / N)$. Then $2^{c}>(N+1) / N$. Hence, in $(X)^{c}$,

$$
\sum_{i<j} x_{i} x_{j}+\sum_{i<j} y_{i} y_{j}=N(N+1) 2^{c}>(N+1)^{2} ;
$$

on the other hand

$$
\sum_{i, j} x_{i} y_{j}=(N+1)^{2}
$$

Thus the $(2 N+2)$-gonal inequality fails in $(X)^{c}$.

Deza [11] proved that $X_{5}$ is $l_{1}$-embeddable if and only if it is pentagonal (=5-gonal). Hence we have the following corollary.

Corollary 1. The metric transform $\left(X_{5}\right)^{c}$ is $l_{1}$-embeddable for $0<c \leq \gamma(2)$, and $\gamma(2)$ is the largest exponent that guarantees $\left(X_{5}\right)^{c}$ to be $l_{1}$-embeddable for all $X_{5}$.

\section{REPEATING NUMBERS}

In a sequence of points, some points may appear repeatedly. Define the maximum repeating number of a sequence as the maximum value of the number of times a point appears in that sequence. For example, the maximum repeating number of the sequence

$$
a, b, c, a, b, b, c, b, c
$$

is 4 provided that $a, b, c$ are all different.

Lemma 3. Suppose that $0<c \leq \gamma(s)$ and $(X)^{c}$ is $(2 N-1)$-gonal. Then, in $(X)^{c}$, the $(2 N+1)$-gonal inequality holds for any $(2 N+1)$-sequence with maximum repeating number at least $2 N /(s+1)$.

Proof. Suppose that $(2)$ is a $(2 N+1)$-sequence with maximum repeating number $r \geq 2 N /(s+1)$.

First, we consider the case $\left\{x_{1}, \ldots, x_{N}\right\} \cap\left\{y_{1}, \ldots y_{N+1}\right\} \neq \varnothing$. Suppose, say, $x_{2}=y_{3}$. Then since the $(2 N-1)$-gonal inequality is valid for

$$
x_{1}, x_{3}, x_{4}, \ldots, x_{N}, y_{1}, y_{2}, y_{4}, \ldots, y_{N+1}
$$

and since

$$
\sum_{i \neq 2} x_{i} x_{2}+\sum_{j \neq 3} y_{j} y_{3}=\sum_{i \neq 2} x_{i} y_{3}+\sum_{j \neq 3} x_{2} y_{j}
$$


the $(2 N+1)$-gonal inequality (1) is valid for the $(2 N+1)$-sequence (2).

Now, suppose that $\left\{x_{1}, \ldots, x_{N}\right\} \cap\left\{y_{1}, \ldots, y_{N+1}\right\}=\varnothing$. Changing suffixes of $x_{i}, y_{j}$ if necessary, we may assume that (3) holds in the sequence (2). Let $k$ be the smallest suffix $i$ such that the maximum repeating number $r$ equals the repeating number of $z_{i}$, where $z_{i}=x_{i}$ or $y_{i}$. In the following we assume that $z_{k}=x_{k}$. (The case $z_{k}=y_{k}$ is similar, and is omitted.) Since $(2 N-1)$-gonal inequality is valid in $(X)^{c}$, we can apply it to the sequence left when $x_{k}$ and $y_{k}$ are removed, to wit

$$
\sum_{\substack{i<j \\ i, j \neq k}} x_{i} x_{j}+\sum_{\substack{i<j \\ i, j \neq k}} y_{i} y_{j} \leq \sum_{i, j \neq k} x_{i} y_{j} .
$$

Hence, in order to prove the $(2 N+1)$-gonal inequality (1) for the sequence (2), it is enough to show that

$$
\sum_{i \in I+J} x_{i} x_{k}+\sum_{j \neq k} y_{j} y_{k} \leq \sum_{i} x_{i} y_{k}+\sum_{j \neq k} x_{k} y_{j}
$$

where

$$
I=\left\{i: i<k, x_{i} \neq x_{k}\right\}, \quad J=\left\{i: i>k, x_{i} \neq x_{k}\right\} .
$$

Note that since $\left\{x_{1}, \ldots, x_{N}\right\} \cap\left\{y_{1}, \ldots, y_{N+1}\right\}=\varnothing$, it follows that $|I|+|J|=$ $N-r$. Now applying Lemma 2 , we have

$$
\begin{aligned}
\sum_{i \in I+J} x_{i} x_{k}+\sum_{j=1}^{N+1} y_{j} y_{k} \leq & \sum_{i \in I}\left\{\left(x_{k} y_{k}\right) / s+x_{k} y_{i}\right\}+\sum_{i \in J}\left\{\left(x_{k} y_{k}\right) / s+x_{i} y_{k}\right\} \\
& +\sum_{j=1}^{k-1}\left\{\left(x_{k} y_{k}\right) / s+x_{j} y_{k}\right\}+\sum_{j=k+1}^{N+1}\left\{\left(x_{k} y_{k}\right) / s+x_{k} y_{j}\right\} .
\end{aligned}
$$

Since $k$ is the minimum value of $i$ such that $x_{i}$ appears $r$ times in the sequence (2), it follows that

$$
I=\{1,2, \ldots, k-1\} .
$$

Hence the right-hand side of the above inequality is

$$
\begin{aligned}
(|I|+ & |J|+N)\left(x_{k} y_{k}\right) / s+\sum_{i \in I+J} x_{i} y_{k}+\sum_{j \neq k} x_{k} y_{j} \\
& =(2 N-r)\left(x_{k} y_{k}\right) / s+\sum_{i \in I+J} x_{i} y_{k}+\sum_{j \neq k} x_{k} y_{j} .
\end{aligned}
$$

Since

$$
\sum_{i} x_{i} y_{k}=r\left(x_{k} y_{k}\right)+\sum_{i \in I+J} x_{i} y_{k}
$$

inequality (5) follows if we show $(2 N-r) / s \leq r$. But this inequality is equivalent to the assumption $r \geq 2 N /(s+1)$.

Theorem 2. The metric transform $\left(X_{2 n}\right)^{c}$ is $(2 n+1)$-gonal for $0<c \leq \gamma(n-1)$. Proof. Suppose $0<c \leq \gamma(n-1)$. Then by Theorem $1,\left(X_{2 n}\right)^{c}$ is $(2 n-1)$ gonal. To prove that $\left(X_{2 n}\right)^{c}$ is $(2 n+1)$-gonal, let $s=n-1, N=n$ in Lemma 
3. Since the maximum repeating number of any $(2 n+1)$-sequence from $\left(X_{2 n}\right)^{c}$ is at least $\lceil(2 n+1) /(2 n)\rceil=2=2 n /(s+1)$, the $(2 n+1)$-gonal inequality holds in $\left(X_{2 n}\right)^{c}$ by Lemma 3 .

Thus $\left(X_{6}\right)^{c}$ is heptagonal (7-gonal) for $0<c \leq \gamma(2)$.

Recently, Avis [5] proved that $X_{6}$ is $l_{1}$-embeddable if and only if it is heptagonal. Hence we have the following corollary.

Corollary 2. The metric transform $\left(X_{6}\right)^{c}$ is $l_{1}$-embeddable for $0<c \leq \gamma(2)$, and $\gamma(2)$ is the largest exponent that guarantees $\left(X_{6}\right)^{c}$ to be $l_{1}$-embeddable for all $X_{6}$.

Theorem 3. The metric transform $\left(X_{n}\right)^{c}$ is hypermetric for $0<c \leq \gamma(n-1)$.

Proof. We show that $\left(X_{n}\right)^{c}$ is $(2 N+1)$-gonal for all $N \geq 1$. This is done by induction on $N$. Since $\left(X_{n}\right)^{c}$ is a metric space, it is 3-gonal by definition. Suppose that $\left(X_{n}\right)^{c}$ is $(2 N-1)$-gonal, $N \geq 2$. Then since the maximum repeating number of any $(2 N+1)$-sequence from $\left(X_{n}\right)^{c}$ is at least $(2 N+1) / n$, the $(2 N+1)$-gonal inequality holds in $\left(X_{n}\right)^{c}$ by Lemma 3 .

\section{EUCLIDEAN EMBEDDINGS}

Since a hypermetric space is of negative type, its "square root" is Euclidean. Hence, from Theorem 3, we have the following.

Corollary 3. The metric transform $\left(X_{n}\right)^{c}$ is Euclidean for $0<c \leq \gamma(n-1) / 2$.

Let $e(n)$ be the supremum of $c$ such that $\left(X_{n}\right)^{c}$ is Euclidean for every $X_{n}$. Since any $n$-point metric space can be isometrically embedded into an $(n+1)$-point metric space, we have $e(n+1) \leq e(n)$. By Blumenthal,

$$
e(4)=1 / 2=\gamma(1) / 2 \text {. }
$$

Using the inequality

$$
\log _{e}(1+1 / s)>1 / s-(1 / s)^{2} / 2>1 /(s+1),
$$

we have

$$
\frac{1}{2} \log _{2}(1+1 /(n-1))>\frac{1}{2}\left(\log _{2} e\right)(1 / n)=0.7213 \ldots / n .
$$

Thus

$$
e(n) \geq 0.7213 \ldots / n \text {. }
$$

By Corollary $1, e(5) \geq \gamma(2) / 2$. But this is the exact value of $e(6)$.

Theorem 4. $e(6)=\gamma(2) / 2=0.2924 \ldots$.

Proof. By Corollary $2,\left(X_{6}\right)^{c}$ is $l_{1}$-embeddable for $0<c \leq \gamma(2)$. Hence $\left(X_{6}\right)^{c}$ is of negative type for that range of $c$, and hence, applying the result of Schoenberg, we have $e(6) \geq \gamma(2) / 2$. On the other hand, if $c>\gamma(2)$ then, by Theorem 1 , there exists a metric space $X_{6}$ whose metric transform $\left(X_{6}\right)^{c}$ is not hexagonal. Therefore, $e(6) \leq \gamma(2) / 2$. 
TABLE 1. Values of $e(n)$ for small $n$

\begin{tabular}{ccccccc}
\hline$n$ & 2 & 3 & 4 & 5 & 6 & 7 \\
$e(n)$ & $\infty$ & 1 & $1 / 2$ & $?$ & $\gamma(2) / 2$ & $?$ \\
\hline
\end{tabular}

Conjecture.

$$
\begin{aligned}
& e(2 n)=\gamma(n-1) / 2, \\
& e(2 n+1)=\gamma(2 n(n+1) /(2 n+1)-1) / 2 .
\end{aligned}
$$

These are true for the known values of $e(n)$. Further, as will be seen in the next section (Theorem 6), if we restrict ourselves to graphs with "truncated distance", the conjecture is also true.

Denote by $i(n)$ the sup of $c$ such that $\left(X_{n}\right)^{c}$ has property (i) for every $X_{n}$. Then it may be also an interesting problem to determine $i(n)$ for such properties as:

(a) $\leq n$-gonal,

(b) hypermetric,

(c) negative type,

(d) $l_{1}$-embeddable.

\section{GRAPHS WITH TRUNCATED DISTANCE}

Here we consider the following problem: Given a graph $G$, up to what value of $c$ is the metric transform $\left(G_{T}\right)^{c}$ Euclidean?

Let $\phi(G ; x)$ be the characteristic polynomial of a graph $G$, that is $\phi(G ; x)=$ $\operatorname{det}(x I-A(G))$, where $A(G)$ is the adjacency matrix of $G$. Define the polynomial $P(G ; x)$ by

$$
P(G ; x)=\phi(G ;-x)-(-1)^{|G|} \phi(\bar{G} ; x-1),
$$

where $\bar{G}$ denote the complement of the graph $G$. Then the following result was proved in Maehara [14]:

A graph $G$ can be embedded in Euclidean space in such a way that adjacent vertices have distance 1 and nonadjacent vertices have distance $t>1$ if and only if

$$
1-1 / t^{2} \leq 1 / z_{\max }
$$

where $z_{\max }$ is the maximum root of the polynomial $P(G ; x)$.

From this result the next follows easily.

Theorem 5. For a graph $G$, the metric transform $\left(G_{T}\right)^{c}$ is Euclidean if and only if $c \leq \frac{1}{2} \gamma\left(z_{\max }-1\right)$.

Proof. The inequality (6) is equivalent to

$$
t \leq\left(1+1 /\left(z_{\max }-1\right)\right)^{1 / 2} .
$$


Since the truncated distances between nonadjacent vertices of $G$ are 2, letting $t=2^{c}$ and applying the above result, we have that $\left(G_{T}\right)^{c}$ is Euclidean if and only if

$$
c \leq \frac{1}{2} \log _{2}\left(1+1 /\left(z_{\max }-1\right)\right)=\gamma\left(z_{\max }-1\right) / 2 .
$$

Corollary 4. For a graph $G$, the truncated metric space $G_{T}$ is of negative type if and only if $z_{\max } \leq 2$.

If $G$ is a regular graph of degree $d$, then the polynomial $P(G)$ is given by

$$
P(G ; x)=(|G| /(x+d)) \phi(G ;-x)
$$

(see [14]). Hence, for a regular graph $G$,

$$
z_{\max }=-(\text { minimum eigenvalue of } G) \text {. }
$$

Corollary 5. For a regular graph $G, G_{T}$ is of negative type if and only if the minimum eigenvalue of $G$ is $\geq-2$.

The graphs with least eigenvalue $\geq-2$ were characterized by Cameron, Goethals, Seidel and Shult [9] in terms of root systems. In the same terms, Terwillinger and Deza [21] gave a characterization of more general class of connected finite distance spaces of negative type [21, Theorem 1] and of hypermetric spaces between them [21, Theorem 2].

Example 1. Let $G$ be the Petersen graph. Then

$$
\phi(G ; x)=(x-3)(x-1)^{5}(x+2)^{4}
$$

(see e.g. [20]), and hence $G_{T}$ is of negative type. Further, since the diameter of $G$ is 2 , we have $G=G_{T}$, and hence the Petersen graph is of negative type.

Example 2. Let $G=K(m, n)$, the complete bipartite graph. Then

$$
\begin{gathered}
\phi(G: x)=\left(x^{2}-m n\right) x^{m+n-2}, \\
\phi(\bar{G} ; x)=(x-m+1)(x+1)^{m-1}(x-n+1)(x+1)^{n-1}
\end{gathered}
$$

(see e.g. [20]), and hence

$$
P(G ; x)=(-x)^{m+n-2}\{(m+n) x-2 m n\} .
$$

Therefore, $z_{\max }=2 m n /(m+n)$. Thus $(G)^{c}=\left(G_{T}\right)^{c}$ is Euclidean if and only if $c \leq \gamma(2 m n /(m+n)-1) / 2$.

Theorem 6. The sup $c_{n}$ of $c$ such that $\left(G_{T}\right)^{c}$ are Euclidean for all n-point graphs $G$ is given by

$$
c_{n}= \begin{cases}\gamma(k-1) / 2 & \text { if } n=2 k, \\ \gamma(2 k(k+1) /(2 k+1)-1) / 2 & \text { if } n=2 k+1 .\end{cases}
$$

Proof. Consider the $2 N$-gonal inequality (1) in the metric transform $\left(G_{T}\right)^{c}$. The left-hand side of $(1)$ does not decrease when the nonzero terms are replaced by $2^{c}$ 's, and the right-hand side of (1) does not increase when the nonzero 
terms are replaced by 1's. Hence the worst case (i.e. the most difficult case for $2 N$-gonal inequality to hold) will be attained by a complete bipartite graph. However, by Example 2, it will be easy to see that the sup of $c$ for $n$-point complete bipartite graphs is given by (7). Hence the theorem follows.

Winkler [23] proved that the graph $G=K_{2}+\bar{K}_{n}$ (= the complement of the disjoint union of $\bar{K}_{2}$ and $K_{n}$ ) is not of negative type for $n>4$ (though $G$ has only one positive eigenvalue). Applying Corollary 4 , let us present a shorter proof of this result.

Example 3 (cf. Winkler [23]). Let $G=K_{2}+\bar{K}_{n}$. Applying Cvetkovic's theorem $[10$, p. 57], we have

$$
\phi\left(K_{2}+\bar{K}_{n} ; x\right)=x^{n-1}\left(x^{3}-(2 n+1) x-2 n\right),
$$

and hence

$$
P(G ; x)=(-x)^{n-1}\left\{-(n+2) x^{2}+2(2 n+1) x-3 n\right\} .
$$

Hence the maximum root is $z_{\max }=3 n /(n+2)$. Thus, if $n>4$, then $z_{\max }>2$, and hence $G \quad\left(=G_{T}\right)$ is not of negative type.

The distance matrix $D$ of $G$ is

$$
\left.\begin{array}{ccccccc}
0 & 1 & 1 & 1 & 1 & \cdots & 1 \\
1 & 0 & 1 & 1 & 1 & \cdots & 1 \\
\hline 1 & 1 & 0 & 2 & 2 & \cdots & 2 \\
1 & 1 & 2 & 0 & 2 & \cdots & 2 \\
\cdot & & & & \cdots & & \\
\cdot & & & & \cdots & & \\
1 & 1 & 2 & 2 & \cdots & 2 & 0
\end{array}\right\} n
$$

Using the spectral resolution

$$
\left(\begin{array}{ccccc}
-x & k & k & \cdots & k \\
k & -x & k & \cdots & k \\
& \cdots & & & \\
& \cdots & & & \\
k & k & \cdots & k & -x
\end{array}\right)=(k n-k-x)(1 / n) J-(x+k)(I-(1 / n) J)
$$

(where $J$ is the matrix with all entries 1 and $I$ is the identity matrix), and the formula

$$
\operatorname{det}\left(\begin{array}{l|l}
M & N \\
\hline P & Q
\end{array}\right)=\operatorname{det}(M) \operatorname{det}\left(Q-P M^{-1} N\right),
$$

the charateristic polynomial of $D$ is calculated as

$$
(-1)^{n}(x+1)(x+2)^{n-1}\left(x^{2}-(2 n-1) x-2\right) .
$$

Thus $G$ has one positive eigenvalue but is not of negative type if $n>4$. 


\section{REFERENCES}

1. M. Aschbacher, P. Bald, E. B. Baum and R. M. Wilson, Embedding of ultrametric spaces in finite dimensional structures, SIAM J. Algebraic Discrete Methods 8 (1987), 564-577.

2. P. Assouad and M. Deza, Espaces metriques plongeables dans un hypercube, Ann. Discrete Math. 8 (1980), 197-210.

3. _ Metric subspaces of $L^{1}$, Publications Mathematiques d'Orsay, 82. 03, Universite de Paris-Sud, 1982.

4. D. Avis, Hypermetric space and Hamming cone, Canad. J. Math. 33 (1981), 795-802.

5. __ All the facets of the six point Hamming cone, Technical Report No. SOCS-88.7 1988.

6. K. Ball, Isometric embedding in $l_{p}$-spaces, European J. Combin. (to appear).

7. L. M. Blumenthal, Remark concerning the euclidean four-point property, Ergebnisse eines Math. Koll. 7 (1936), 8-10.

8. __ Theory and applications of distance geometry, Chelsea, New York, 1970.

9. P. J. Cameron, J. M. Goethals, J. J. Seidel and E. E. Shult, Line graphs, root systems and elliptic goemetry, J. Algebra 43 (1976), 305-327.

10. D. Cvetkovic, M. Doob and H. Sachs, Spectra of graphs, Academic Press, New York, 1980.

11. M. Deza (as M. E. Tylkin), On Hamming geometry of unitary cubes, Dokl. Akad. Nauk SSR 134 (1960), 1037-1040.

12. R. L. Graham and P. M. Winkler, On isometric embeddings of graphs, Trans. Amer. Math. Soc. 288 (1985), 527-536.

13. J. B. Kelly, Hypermetric spaces, Lecture Notes in Math., Vol. 490, Springer-Verlag 1975, pp. 17-31.

14. H. Maehara, Regular embeddings of a graph, Pacific J. Math. 107 (1983), 393-402.

15. $\frac{1}{246}$, Metric transforms of finite spaces and connected graphs, Discrete Math. 61 (1986), 235-

16. J. von Neumann and I. J. Schoenberg, Fourier integrals and metric geometry, Trans. Amer. Math. Soc. 50 (1941), 226-251.

17. R. P. Roth and P. M. Winkler, Collapse of the metric hierarchy for bipartite graphs, European J. Combin. 7 (1986), 371-375.

18. I. J. Schoenberg, Remarks to Maurice Frechet's article, Ann. of Math. (2) 36 (1935), 724-732.

19. __, Metric space and completely monotone functions, Ann. of Math. (2) 39 (1938), 811-841.

20. A. J. Schewenk and R. J. Wilson, On the eigenvalue of a graph, Selected Topics in Graph Theory (L. W. Beineke and R. J. Wilson, eds.), Academic Press, New York, 1978.

21. P. Terwillinger and M. Deza, The classification of finite connected hypermetric spaces, Graphs and Combinatorics 3 (1987), 293-298.

22. W. A. Wilson, On certain types of continuous transformations of metric spaces, Amer. J. Math. 57 (1935), 62-68.

23. P. M. Winkler, On graphs which are metric spaces of negative type, Graph Theory with Application to Algorithms and Computer Science (Y. Alavi et. al., eds.), Wiley, New York, 1985, pp. 801-810.

24. H. S. Witsenhausen, Minimum dimension embedding of finite metric spaces, J. Combin. Theory Ser. A 42 (1986), 184-199.

CNRS, Paris, France

Department of Mathematics, Ryukyu University, Okinawa, Japan 